\begin{tabular}{|c|l|}
\hline Title & Response of shear stress to ac electric fields under steady shear flow in a droplet-dispersed phase \\
\hline Author(s) & Na, Y ang Ho; A ida, Kohei; Sakai, Ryosuke; Kakuchi, Toy oji; Orihara, Hiroshi \\
\hline Citation & $\begin{array}{l}\text { Physical Review E, 80(6), 061803 } \\
\text { https://doi.org/10.1103/PhysRevE.80.061803 }\end{array}$ \\
\hline Issue Date & 2009-12 \\
\hline Doc URL & http://hdl.handle.net/2115/42550 \\
\hline Rights & ○2009 The A merican Physical Society \\
\hline Type & article \\
\hline File Information & PRE80-6_061803.pdf \\
\hline
\end{tabular}

Instructions for use 


\title{
Response of shear stress to ac electric fields under steady shear flow in a droplet-dispersed phase
}

\author{
Yang Ho Na, Kohei Aida, Ryosuke Sakai, Toyoji Kakuchi, and Hiroshi Orihara* \\ Graduate School of Engineering, Hokkaido University, Sapporo 060-8628, Japan \\ (Received 26 May 2009; revised manuscript received 28 September 2009; published 30 December 2009)
}

\begin{abstract}
We have measured the response of shear stress to ac electric fields under steady shear flow in the dropletdispersed phase of an immiscible polymer blend. A characteristic mode was found under steady shear flow, the relaxation frequency of which increased with increasing the shear rate. In the frequency dispersion, a scaling relation derived from dimensional analysis was confirmed to hold. The origin of the mode was investigated on the basis of the Maffettone-Minale (MM) model, in which the droplet shape is described by a second-rank tensor. The frequency dispersion of the response was also calculated using a modified MM model.
\end{abstract}

DOI: 10.1103/PhysRevE.80.061803

PACS number(s): 83.80.Tc, 83.50.-v, 83.80.Gv, 83.80.Iz

\section{INTRODUCTION}

Frequently, immiscible liquid blends (emulsions) show a droplet-dispersed phase. The spherical droplets are easily deformed to ellipsoidal ones by small external fields, and they relax back to the spheres by interfacial tension after removal of the fields. In droplet-dispersed phases, the rheological properties strongly depend on the droplet shape and size. Therefore, they exhibit complicated rheological responses to external fields. When the field is a shear stress, the response is characterized by the complex modulus. In oscillatory measurements, a characteristic relaxation appears at low frequencies in the storage modulus due to the interfacial tension [1-4]. The relaxation was theoretically investigated by Palierne and an excellent agreement was obtained with the experiments $[2,4]$.

Viscoelastic measurements are usually made at equilibrium with applying a small oscillating shear flow as a perturbation. Recently, on the other hand, oscillation measurements under steady shear flow have been performed $[5,6]$. Under steady shear flow, spherical droplets are elongated to change into ellipsoidal ones. Superimposition of a small oscillating shear flow as a perturbation causes the deformation and reorientation of ellipsoidal droplets, which can be detected through the stress change. In the measurements, it was observed that the storage modulus becomes negative under steady shear flow [6]. The result was analyzed on the basis of the Maffettone-Minale (MM) [7], which was confirmed to be valid for ordinary oscillation measurements [8]. Here, it should be emphasized that the system is not at equilibrium but at steady state. From this point of view, the superposition measurements will become a useful method to investigate the fluctuations at nonequilibrium steady state, which has interesting features [5,6,9-12].

When two immiscible liquids have a mismatch of electrical properties, such as permittivity and conductivity, the droplets can respond to electric fields. In some blends, it was observed that the droplets are elongated along the electric field $[13,14]$. The response is dependent on the electrical properties and the frequency for ac electric fields, which was investigated in detail by Torza et al. [13] Also under steady

\footnotetext{
*orihara@eng.hokudai.ac.jp
}

shear flow, the deformation and reorientation of ellipsoidal droplets may be induced by application of electric fields, which can be detected through the stress change. This suggests that the fluctuations at steady state can be measured by applying an oscillating electric field as well as the shear flow used in the superposition measurement.

In this paper, we measured the shear stress response of an immiscible polymer blend to ac electric fields under steady shear flow. First, the results are discussed on the basis of a scaling relation derived from dimensional analysis. Then, the normal modes of droplet deformation under steady shear flow are investigated to clarify a feature characteristic to our nonequilibrium steady system in terms of the MM model. The frequency dependence of the shear stress is also calculated using the MM model with an additional term related to electrical stress.

\section{EXPERIMENT}

A liquid-crystalline polymer (LCP) and a polydimethylsiloxane (DMS) were used as fluids. The LCP was synthesized according to the method of Ref. [15]. The DMS (KF96H10000) was purchased from Shin-Etsu Chemical (Tokyo, Japan). All the experiments were done at $25^{\circ} \mathrm{C}$, where the LCP was in the isotropic phase. It should be noted that electrorheological effect is not directly related to the orientational change in mesogens, which play a role to enhance the dielectric permittivity. The viscosities, relative dielectric constants, and conductivities of the LCP and DMS are 19.5 $\mathrm{Pa}$ s, $10.0 \mathrm{~Pa}$ s, 15.2, 2.8, $1.0 \times 10^{-8} \Omega^{-1} \mathrm{~m}^{-1}$, less $10^{-12} \Omega^{-1} \mathrm{~m}^{-1}$, respectively. The viscosities were measured by a rheometer (Physica MCR300, Anton Paar) and the relative dielectric constants and conductivities by a dynamic ion density measurement system (MTR1, Toyo corporation). We prepared a blend of LCP:DMS $=1: 5$ in weight. This mixture was prepared by dissolving them in tetrahydrofuran followed by evaporation of the solvent under vacuum. The blend was sandwiched between a bottom glass plate with an indium-tinoxide-coated electrode and a rotating metal disk of the rheometer. As previously described [16,17], measurements were carried out with the parallel-plate rotational rheometer. A schematic picture of the system is shown in Fig. 1(a). The diameter of the rotating plate and the gap between the two parallel plates were 35 and $0.2 \mathrm{~mm}$, respectively. Since we 

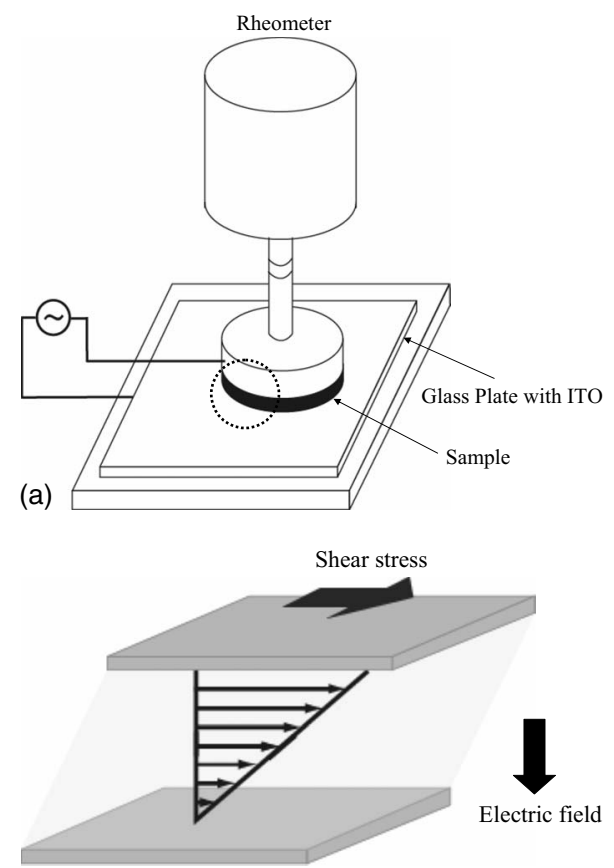

(b)

FIG. 1. (a) Schematic illustration of the system used in the measurement and (b) the flow direction and electric field in the system.

used a parallel-plate rotational viscometer and the shear rate depends on its position, the shear rate was defined as the one at the periphery of the upper disk. The shear stress at the edge of the top plate was calculated from the torque by assuming that the fluid is Newtonian, though it is a crude approximation in our fluids. Figure 1(b) shows the relation between the flow direction, the velocity gradient, and the electric field. Electric fields were applied to the blend by using a synthesizer (Model 1940, NF Electric Instruments) and a high-voltage amplifier (Model 609C-6, Trek).

\section{RESULTS AND DISCUSSION}

Dynamics of the deformed droplets under steady shear flow was investigated by the application of an ac electric field. When an ac electric field with an amplitude $E_{0}$ and an angular frequency $\omega, E=E_{0} \cos \omega t$, is applied, the shear stress may be written as

$$
\sigma(t)=\sigma_{0}+\sigma_{2,0}(\omega)+\operatorname{Re}\left[\sigma_{2,2}(\omega) e^{i 2 \omega t}\right],
$$

where $\sigma_{0}$ is the shear stress without electric field. Note that the stress is independent of the polarity of the electric field because the fluid is nonpolar and, therefore, the $2 \omega$ response appears and $\sigma_{2,0}(\omega)$ and $\sigma_{2,2}(\omega)$ should be proportional to the square of $E_{0}$ for a small $E_{0}$. Figure 2 shows the dependence of $\left|\sigma_{2,2}(\omega)\right|$ on the square of $E_{0}$ at $\dot{\gamma}=20 \mathrm{~s}^{-1}$ and $\omega$ $=1.38 \mathrm{rad} \mathrm{s}^{-1}$. All the measurements were made in the range that the proportionality holds. Figure 3 shows the frequency dependences of the real part $\sigma_{2,2}^{\prime}$ and the imaginary part $\sigma_{2,2}^{\prime \prime}$ of $\sigma_{2,2}=\sigma_{2,2}^{\prime}-i \sigma_{2,2}^{\prime \prime}$ measured with a steady shear flow of $20 \mathrm{~s}^{-1}$. We have a characteristic frequency dispersion different from the Debye-type relaxation. Especially, the real part

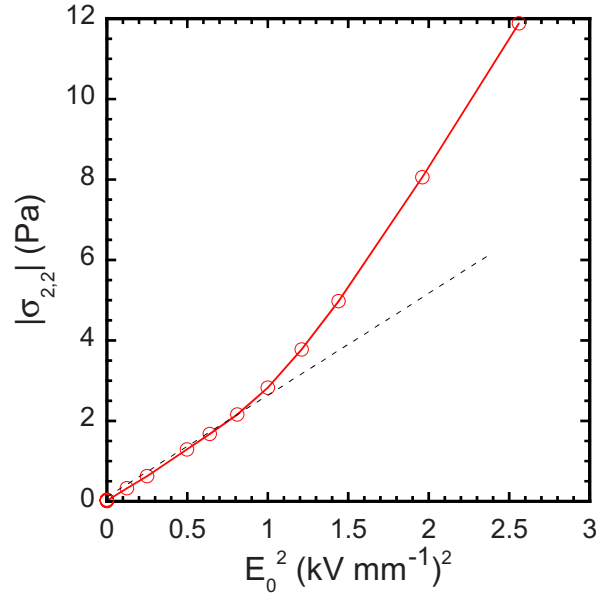

FIG. 2. (Color online) Dependence of $\left|\sigma_{2,2}(\omega)\right|$ on the squared amplitude of ac electric field $E_{0}^{2}$, at $\dot{\gamma}=20 \mathrm{~s}^{-1}$ and $\omega$ $=1.38 \mathrm{rad} \mathrm{s}^{-1}$.

becomes negative. However, it should be noted that the negative part depends on the shear rate, as shown later. Before investigating the details of the dispersion, we will discuss the scaling property of $\sigma_{2,2}(\omega)$. Scaling properties of blends have already been investigated under a constant and step electric field $[18,19]$.

As has been mentioned, $\sigma_{2,2}(\omega)$ should be proportional to the square of $E_{0}$. Taking this into account and applying dimensional analysis, we obtain

$$
\sigma_{2,2}(\omega)=\varepsilon_{1} E_{0}^{2} f\left(\omega / \dot{\gamma}, \eta_{1} / \eta_{2}, \varepsilon_{1} / \varepsilon_{2}, \kappa_{1} / \kappa_{2}, \omega \tau_{c}, \varphi\right),
$$

with [19]

$$
\tau_{c}=\frac{\varepsilon_{1}+2 \varepsilon_{2}}{\kappa_{1}+2 \kappa_{2}},
$$

where $\eta_{1}$ and $\eta_{2}$ are the viscosities of the droplet and matrix phases, and $\varepsilon_{1}$ and $\varepsilon_{2}$ are the dielectric constants, $\kappa_{1}$ and $\kappa_{2}$ are the conductivities, and $\varphi$ is the volume fraction, respectively. One might think that the droplet size $R$ should also be

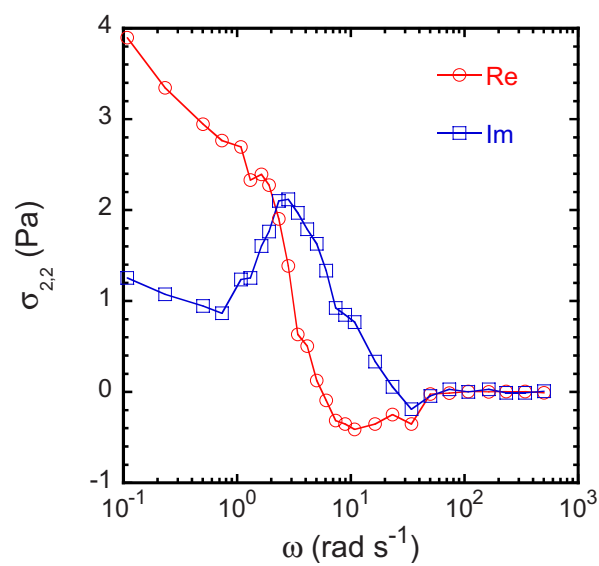

FIG. 3. (Color online) Frequency dependences of the real part $\sigma_{2,2}^{\prime}$ and the imaginary $\sigma_{2,2}^{\prime \prime}$ of $\sigma_{2,2}(\omega)=\sigma_{2,2}^{\prime}-i \sigma_{2,2}^{\prime \prime}$ measured under a steady shear flow of $20 \mathrm{~s}^{-1}$ and an ac electric field of $E_{0}$ $=0.90 \mathrm{kV} \mathrm{mm}^{-1}$. 
included, which is not a parameter given externally but determined by the kinetics of the system from the shear rate, viscosities, and interfacial tension $\Gamma$ in the steady state [20]. Interestingly, however, there appears no interfacial tension in Eq. (2). This is because one can construct neither quantity with unit of stress nor dimensionless quantity including $\Gamma . \tau_{c}$ in Eq. (3) is the relaxation time of charge in a droplet. For $\omega \ll \tau_{c}^{-1}$, the charge relaxation occurs, while for $\omega \gg \tau_{c}^{-1}$, it cannot do. Therefore, in general, the deformation of droplets becomes small at frequencies higher than $\tau_{c}^{-1}$. On the other hand, the deformed shape (oblate or prolate) also depends on the electrical properties, the frequency of the applied field, and the viscosities [13]. In our blend, droplets are elongated along the applied field to become prolate. At angular frequencies far from $\omega_{c}=\tau_{c}^{-1}, \sigma_{2,2}(\omega)$ in Eq. (2) may be independent of $\omega$ through $\omega \tau$ but dependent on $\omega$ through $\omega / \dot{\gamma}$. This indicates that the plot of $\sigma_{2,2}(\omega) / E_{0}^{2}$ against $\omega / \dot{\gamma}$ becomes a universal curve for $\omega \ll \omega_{c}$ or $\omega \gg \omega_{c}$. In our blend $\tau_{c} \approx 0.02 \mathrm{~s}$, that is, $\omega_{c} \approx 50 \mathrm{rad} \mathrm{s}^{-1}$. In Fig. 3 , therefore, we may apply this scaling relation for $\omega<50 \mathrm{rad} \mathrm{s}^{-1}$.

Here, we should mention that in the parallel-plate rheometer, the shear rate depends on the position; in the above scaling relation, it was assumed to be constant. However, it can be shown that the scaling relation is still valid in our case as well. For the parallel-plate rheometer, we usually define the shear rate as the one at the edge of the rotating disk and the shear stress there is calculated from the measured torque by assuming that the fluid is Newtonian. It is easily shown that the shear stress thus defined $\sigma_{2,2}^{(p)}$ obeys the following relation similar to Eq. (2):

$$
\sigma_{2,2}^{(p)}=\varepsilon_{1} E_{0}^{2} F\left(\omega / \dot{\gamma}_{p}, \eta_{1} / \eta_{2}, \varepsilon_{1} / \varepsilon_{2}, \kappa_{1} / \kappa_{2}, \omega \tau_{c}\right),
$$

with

$$
\begin{aligned}
& F\left(x, \eta_{1} / \eta_{2}, \varepsilon_{1} / \varepsilon_{2}, \kappa_{1} / \kappa_{2}, \omega \tau\right) \\
& \quad \equiv 4 x^{3} \int_{0}^{x^{-1}} p^{2} f\left(p^{-1}, \eta_{1} / \eta_{2}, \varepsilon_{1} / \varepsilon_{2}, \kappa_{1} / \kappa_{2}, \omega \tau_{c}\right) d p,
\end{aligned}
$$

where $\dot{\gamma}_{p}$ is the shear rate at the edge. For simplicity, we will omit the subscript " $p$ " hereafter.

Figure 4 shows a plot of $\sigma_{2,2}(\omega) / \varepsilon_{1} E_{0}^{2}$ vs $\omega / \dot{\gamma}$ for different $\dot{\gamma}$, where $E_{0}$ was set for each $\dot{\gamma}$ so that a dimensionless number $M_{n}$, which is similar to the Mason number [21],

$$
M_{n}=\eta_{1} \dot{\gamma} / \varepsilon_{1} E_{0}^{2}
$$

is constant. This ensures that the ratio $\sigma_{2,2}(\omega) / \sigma_{0}$ is independent of the set of $E_{0}$ and $\dot{\gamma}$ thus determined, which was necessary to have an enough precision for $\sigma_{2,2}(\omega)$ in each measurement. All the measurements were carried out under the condition of $M_{n}=3.2$. As is seen from Fig. 4, all the data almost fall on a universal curve, though they are scattered. However, there is a small systematic deviation: the position of the minimum in the real part moves from $\omega / \dot{\gamma} \approx 0.2$ (at $\dot{\gamma}=60 \mathrm{~s}^{-1}$ ) to higher frequencies, and eventually the minimum almost vanishes for $\dot{\gamma}=5 \mathrm{~s}^{-1}$. The reason for the deviation is not yet clarified at present.

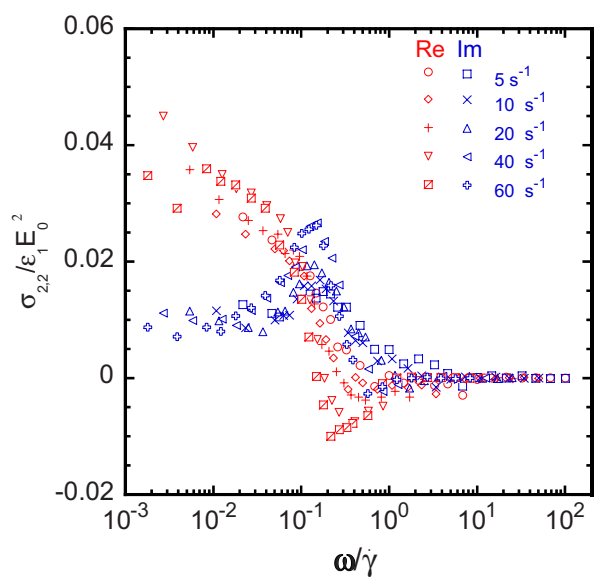

FIG. 4. (Color online) Plot of $\sigma_{2,2}(\omega) / \varepsilon_{1} E_{0}^{2}$ vs $\omega / \dot{\gamma}$ for $\dot{\gamma}=5,10$, 20, 40, and $60 \mathrm{~s}^{-1}$, where $E_{0}$ was set for each $\dot{\gamma}$ so that $M_{n}=3.2$.

Next, we turn our attention to the dispersion in Fig. 3, which has the following characteristics: it is not simple relaxation (Debye type) and the real part becomes negative at high frequencies for large shear rates, i.e., it has an oscillatory character. We discuss it on the basis of the MM model [7]. Under a constant shear flow, droplets are elongated to become ellipsoidal. In addition, when subjected to an ac electric field with an angular frequency $\omega$, the ellipsoid may rotate around the vorticity axis and may expand and shrink in the directions of all the semiaxes, preserving the droplet volume. These changes modify the shear stress with the angular frequency $2 \omega$. The response of droplet to the electric field can be clarified by investigating the normal modes in the steady state. Hereafter, we will briefly review the MM model.

In the MM model, it is assumed that the droplet is always ellipsoidal and the shape is described in terms of a symmetric second-rank tensor $\mathbf{S}$ with eigenvalues representing the squared semiaxes of the ellipsoid. The temporal evolution of $\mathbf{S}$ is given as

$$
\frac{d \mathbf{S}}{d t}-\boldsymbol{\Omega} \cdot \mathbf{S}+\mathbf{S} \cdot \boldsymbol{\Omega}=-\frac{f_{1}}{\tau}[\mathbf{S}-g(\mathbf{S}) \mathbf{I}]+f_{2}(\mathbf{A} \cdot \mathbf{S}+\mathbf{S} \cdot \mathbf{A}),
$$

with

$$
\tau=\eta_{2} R / \Gamma,
$$

where $\tau$ is a characteristic time, $\mathbf{I}$ is the second-rank unit tensor, $\mathbf{A}$ and $\boldsymbol{\Omega}$ are the symmetrical and antisymmetrical parts of the velocity gradient tensor $\nabla \mathbf{v}$,

$$
\begin{aligned}
& \mathbf{A}=\frac{1}{2}\left(\boldsymbol{\nabla} \mathbf{v}+\boldsymbol{\nabla} \mathbf{v}^{\mathrm{T}}\right), \\
& \boldsymbol{\Omega}=\frac{1}{2}\left(\boldsymbol{\nabla} \mathbf{v}-\boldsymbol{\nabla} \mathbf{v}^{\mathrm{T}}\right) .
\end{aligned}
$$

The dimensionless coefficients $f_{1}$ and $f_{2}$ are given as

$$
f_{1}=\frac{40(\lambda+1)}{(2 \lambda+3)(19 \lambda+16)},
$$




$$
f_{2}=\frac{5}{2 \lambda+3},
$$

where $\lambda=\eta_{1} / \eta_{2}$ is the viscosity ratio. The function $g(\mathbf{S})$ is introduced to preserve the droplet volume and is given as

$$
g(\mathbf{S})=3 \frac{I_{3}(\mathbf{S})}{I_{2}(\mathbf{S})},
$$

where $I_{3}(\mathbf{S})$ is the third invariant of $\mathbf{S}$, i.e., its determinant, and $I_{2}(\mathbf{S})$ is the second invariant defined as

$$
I_{2}(\mathbf{S})=\frac{1}{2}\left[\{\operatorname{Tr} \mathbf{S}\}^{2}-\operatorname{Tr} \mathbf{S}^{2}\right] .
$$

For a simple shear flow, $\mathbf{A}$ and $\boldsymbol{\Omega}$ become

$$
\mathbf{A}=\frac{1}{2}\left(\begin{array}{ccc}
0 & \dot{\gamma} & 0 \\
\dot{\gamma} & 0 & 0 \\
0 & 0 & 0
\end{array}\right), \quad \boldsymbol{\Omega}=\frac{1}{2}\left(\begin{array}{ccc}
0 & \dot{\gamma} & 0 \\
-\dot{\gamma} & 0 & 0 \\
0 & 0 & 0
\end{array}\right) .
$$

In the steady state of $d \mathbf{S} / d t=0$ in Eq. (7), let $L, B$, and $W$ be the three semiaxes of the ellipsoid, where $L$ and $B$ are in the $x_{1}-x_{2}$ shear plane $(L>B)$ and $W$ along the vorticity $\left(x_{3}\right.$ axis), and define the orientation angle $\theta$ between the $x_{1}$ and $L$ axes. From Eq. (7), these quantities are given [7] as

$$
\begin{gathered}
\tan 2 \theta=\frac{f_{1}}{\dot{\gamma} \tau}, \\
W^{2}=\left[1-\frac{f_{2}^{2}}{1+f_{1}^{2} /(\dot{\gamma} \tau)^{2}}\right]^{1 / 3} R^{2}, \\
L^{2}=\frac{f_{1}}{f_{1}-f_{2} \dot{\gamma} \tau \sin 2 \theta} W^{2}, \\
B^{2}=\frac{f_{1}}{f_{1}+f_{2} \dot{\gamma} \tau \sin 2 \theta} W^{2},
\end{gathered}
$$

where $R$ is the radius without shear flow.

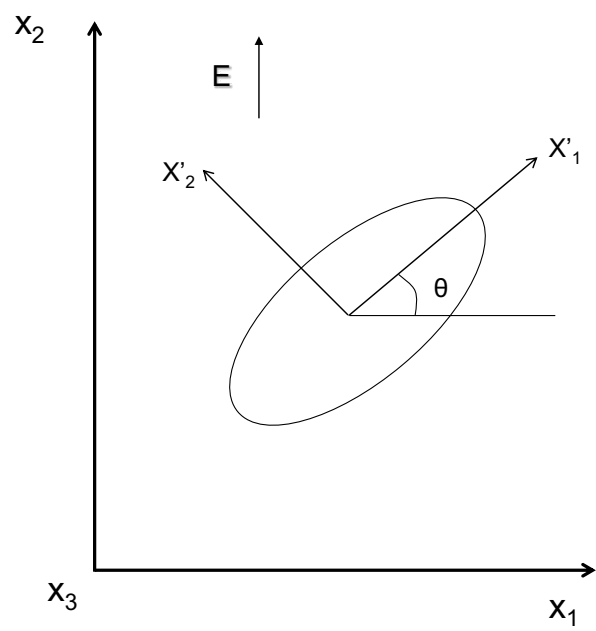

FIG. 5. Illustration of the $x_{1}^{\prime}-x_{2}^{\prime}-x_{3}$ coordinate system with the semiaxes of the ellipsoid and the electric field.

Now, we calculate the normal mode. We can write a $\mathbf{S}^{\prime}$ tensor including small fluctuations from the steady state in the $x_{1}^{\prime}-x_{2}^{\prime}-x_{3}$ coordinate system with the semiaxes of the ellipsoid (Fig. 5),

$$
\mathbf{S}^{\prime}=\left(\begin{array}{ccc}
L^{2} & 0 & 0 \\
0 & B^{2} & 0 \\
0 & 0 & W^{2}
\end{array}\right)+\left(\begin{array}{ccc}
\delta S_{1} & \delta S_{12} & \delta S_{13} \\
\delta S_{12} & \delta S_{2} & \delta S_{23} \\
\delta S_{13} & \delta S_{23} & \delta S_{3}
\end{array}\right) .
$$

From Eq. (7), we obtain the evolution equations for the fluctuations,

$$
\begin{aligned}
\frac{d}{d t}\left(\begin{array}{c}
\delta S_{1} \\
\delta S_{2} \\
\delta S_{3} \\
\delta S_{12}
\end{array}\right) & =\mathbf{M}_{1}\left(\begin{array}{c}
\delta S_{1} \\
\delta S_{2} \\
\delta S_{3} \\
\delta S_{12}
\end{array}\right), \\
\frac{d}{d t}\left(\begin{array}{c}
\delta S_{13} \\
\delta S_{23}
\end{array}\right) & =\mathbf{M}_{2}\left(\begin{array}{l}
\delta S_{13} \\
\delta S_{23}
\end{array}\right)
\end{aligned}
$$

with

$$
\mathbf{M}_{1}=\left(\begin{array}{cccc}
f_{1} / \tau\left(J_{1}-1\right)+f_{2} \dot{\gamma} \sin 2 \theta & f_{1} / \tau J_{2} & f_{1} / \tau J_{3} & \left(f_{2} \cos 2 \theta+1\right) \dot{\gamma} \\
f_{1} / \tau J_{1} & f_{1} / \tau\left(J_{2}-1\right)-f_{2} \dot{\gamma} \sin 2 \theta & f_{1} / \tau J_{3} & \left(f_{2} \cos 2 \theta-1\right) \dot{\gamma} \\
f_{1} / \tau J_{1} & f_{1} / \tau J_{2} & f_{1} / \tau\left(J_{3}-1\right) & 0 \\
\left(f_{2} \cos 2 \theta-1\right) \dot{\gamma} / 2 & \left(f_{2} \cos 2 \theta+1\right) \dot{\gamma} / 2 & 0 & -f_{1} / \tau
\end{array}\right),
$$

$$
\mathbf{M}_{2}=\left(\begin{array}{cc}
-f_{1} / \tau+f_{2} \dot{\gamma} \sin 2 \theta / 2 & \left(f_{2} \cos 2 \theta+1\right) \dot{\gamma} / 2 \\
\left(f_{2} \cos 2 \theta-1\right) \dot{\gamma} / 2 & -f_{1} / \tau-f_{2} \dot{\gamma} \sin 2 \theta / 2
\end{array}\right),
$$

$$
J_{1}=\frac{W^{4}}{3 R^{6}}\left\{2 B^{2}-W^{2}\right\},
$$

where

$$
J_{2}=\frac{W^{4}}{3 R^{6}}\left\{2 L^{2}-W^{2}\right\},
$$




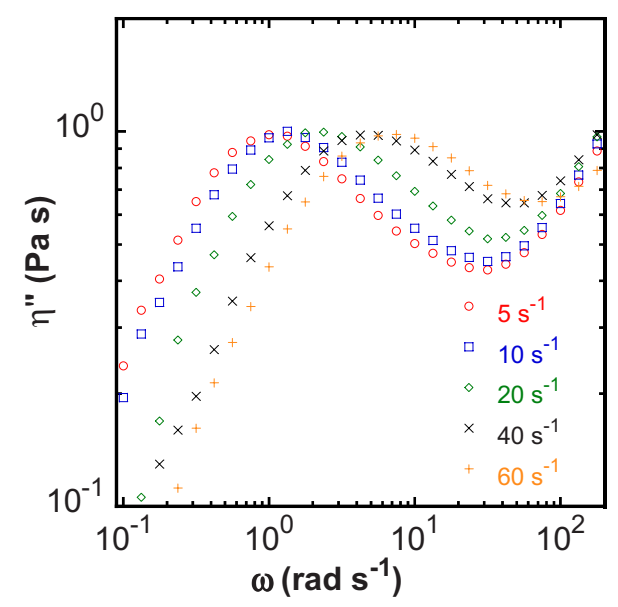

FIG. 6. (Color online) Frequency dispersions of out-of-phase viscosity for the LCP/DMS blend after applying preshears of 5, 10, 20,40 , and $60 \mathrm{~s}^{-1}$, respectively.

$$
J_{3}=\frac{W^{2}}{3 R^{6}}\left\{3 L^{2} B^{2}-W^{2}\left(L^{2}+B^{2}\right)\right\} .
$$

From the matrix $\mathbf{M}_{1}$, we can obtain two deformation modes preserving the volume and one orientation mode around the $x_{3}$ axis, which are mixed to construct normal modes. From $\mathbf{M}_{2}$, on the other hand, two orientation modes can be derived around the $x_{1}^{\prime}$ and $x_{2}^{\prime}$ axes. Especially at $\dot{\gamma}=0$, the eigenvalues of $\mathbf{M}_{1}$ are $-f_{1} / \tau$ and 0 . The modes corresponding to the former eigenvalue are triply degenerated; two independent deformation modes and one orientation mode around the $x_{3}$ axis. The other mode corresponding to zero eigenvalue violates the constant volume condition and so it should be omitted.

For $\dot{\gamma} \neq 0$, it is readily seen that the matrixes (23) and (24) become asymmetrical, implying that the eigenvalues can be complex. For complex eigenvalues, the corresponding normal modes can oscillate. Therefore, it is thought that the negative real part of the dispersion may come from the modes with complex eigenvalues. On the other hand, the complex eigenvalues may bring about damped oscillations in time dependence. Actually, they were shown by numerical calculations based on the Navier-Stokes equations [22]. In our experiments, the complex eigenvalues of the matrix $\mathbf{M}_{1}$ may be responsible for the negative real part because applied electric fields can excite only the modes related to $\mathbf{M}_{1}$ from the symmetry. Let us calculate the eigenvalues in our experiment. Since in our blend, the droplet size and the interfacial tension were not measured, we determined $f_{1} / \tau$ in Eq. (23) from the oscillation measurement without electric field after applying a steady shear flow. Figure 6 shows the frequency dispersions of the imaginary part of the complex viscosity at several shear rates. A relaxation due to the interface of dispersed droplets is clearly seen at each shear rate [4]. As mentioned above, the peak frequency should be equal to $f_{1} / \tau$ in the MM model [8]. The relaxation frequency $\omega_{\text {rel }}=f_{1} / \tau$ thus obtained is shown in Fig. 7, which is seen to be proportional to the shear rate $\dot{\gamma}$. This result indicates that the droplet size $R$ is inversely proportional to $\dot{\gamma}$ because $\omega_{\text {rel }}=f_{1} / \tau$

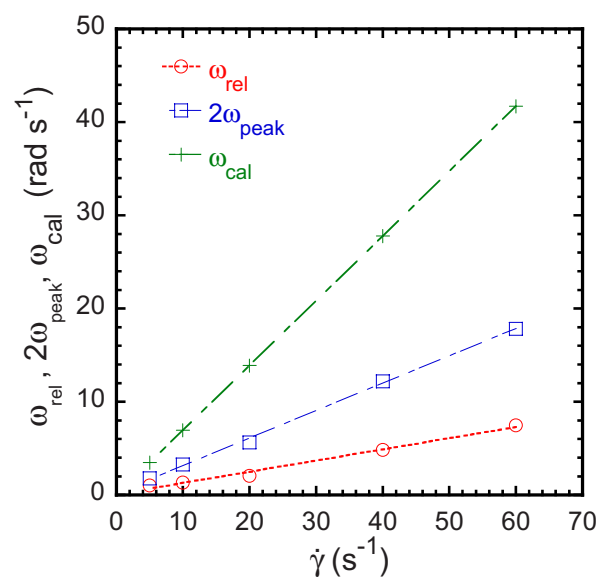

FIG. 7. (Color online) Dependences of $\omega_{\text {rel }}, 2 \omega_{\text {peak }}$, and $\omega_{\text {cal }}$ on the shear rate. These are proportional to the shear rate.

$=f_{1} \Gamma /\left(\eta_{2} R\right)$ from Eq. (8). Note that this is easily derived from dimensional analysis $R \propto \Gamma /\left(\eta_{2} \dot{\gamma}\right)$. For example, at $\dot{\gamma}$ $=20 \mathrm{~s}^{-1}, \omega_{\text {rel }}=f_{1} / \tau$ is about $2.5 \mathrm{rad} \mathrm{s}^{-1}$. From Eq. (16), we obtain the orientation angle $\theta$ as $3.6^{\circ}$ and, furthermore, from Eqs. (17)-(19), the aspect ratio $L: B: W=1: 0.40: 0.53$. It should be noted that the angle and ratio are independent of the shear rate because $\omega_{\text {rel }} \propto \dot{\gamma}$, i.e., $\dot{\gamma} \tau$ is constant in Eqs. (16)-(19). For $\omega_{\text {rel }}=2.5 \mathrm{rad} \mathrm{s}^{-1}$, the matrix $\mathbf{M}_{1}$ has eigenvalues; $-2.0 \pm 13.9 i \mathrm{rad} \mathrm{s}^{-1},-2.5 \mathrm{rad} \mathrm{s}^{-1}$. Note that $-f_{1} / \tau$ is always one of the eigenvalues. The complex eigenvalues may be responsible for our characteristic dispersions and the imaginary part may correspond to the eigenfrequency of the mode. However, the imaginary part of $13.9 \mathrm{rad} \mathrm{s}^{-1}$ is larger than estimated from the experiment. The eigenfrequency may be equal to twice the peak frequency in Fig. 3 because the Maxwell stress acting on droplets is proportional to the square of the applied electric field and so the stress should oscillate at $2 \omega$. The eigenfrequency $\left(2 \omega_{\text {peak }}\right.$, where $\omega_{\text {peak }}$ is the peak frequency) is about $6 \mathrm{rad} \mathrm{s}^{-1}$ from Fig. 3, which is considerably smaller than $13.9 \mathrm{rad} \mathrm{s}^{-1}$. The same results were obtained for the other shear rates. The dependences of the measured eigenfrequency $2 \omega_{\text {peak }}$ and the calculated one (the imaginary part) $\omega_{\text {cal }}$ on the shear rate are shown in Fig. 7. The frequency $2 \omega_{\text {peak }}$ is proportional to the shear rate, indicating that the droplet size should be inversely proportional to the shear rate under ac electric fields as well as under no field. The calculated eigenfrequencies are almost twice as large as the measured ones for all the shear rates.

The discrepancy can be reduced by taking into account the hydrodynamic effect and by taking a different choice of $f_{2}$. First, let us consider the former, which decreases the net shear rate at a droplet. We estimated the effect adopting the method used by Chen et al. [6] From the results obtained by Frankel and Acrivos [23], they calculated the velocity gradient around an isolated droplet subjected to a simple shear flow by assuming that the droplet was not deformed and estimated the shear rate around it, which is disturbed by the existence of the droplet and strongly depends on the distance from the droplet. From the volume fraction of our blend $\varphi$ $=1 / 6$, we obtain the mean nearest-neighbor distance between droplets as $l_{m}=2.5 R$ [24], and then the shear rate change is 
given as $\Delta \dot{\gamma}=-0.3 \dot{\gamma}$, using an equation derived by Chen $e t$ al. [6] For example, when the applied shear rate is $20 \mathrm{rad} \mathrm{s}^{-1}$, the shear rate at a droplet may be $14 \mathrm{rad} \mathrm{s}^{-1}$. For this shear rate, the eigenvalues become $-2.1 \pm 9.7 i$, giving a better agreement. Next, we discuss the choice of $f_{2}$. Actually, in the MM theory a modification of $f_{2}$ was made to have better results at high viscosity and high Capillary number. Another choice of $f_{2}$ is given as [7]

$$
f_{2}=\frac{5}{2 \lambda+3}+\frac{3 C_{a}^{2}}{2+6 C_{a}^{2+\delta}} \frac{1}{1+\varepsilon \lambda^{2}},
$$

where $C_{a}$ is the Capillary number, $\delta$ and $\varepsilon$ are small positive numbers introduced to preserve the $\lambda^{-1}$ and the affine motion limit, respectively. When $\delta=\varepsilon=0$, we have the maximum of $f_{2}$. In our experiments, the Capillary number is obtained using the relation $C_{a}=\left(f_{1} / \tau\right)^{-1} f_{1} \dot{\gamma}$, where $f_{1} / \tau=2.5 \mathrm{~s}^{-1}, f_{1}$ $=0.322$ from Eq. (11), and $\dot{\gamma}=20 \mathrm{~s}^{-1}$, giving $C_{a}=2.58$. Substitution of this value into Eq. (28) yields $f_{2}=1.2$. When we use this value instead of the one (0.725) obtained from Eq. (11), we could obtain no positive real value for Eq. (17). However, better results were obtained using values larger than 0.725. As an example, for $f_{2}=0.9$ we have a positive real value for Eq. (17) and eigenvalues of $-1.8 \pm 6.3 i \mathrm{rad} \mathrm{s}^{-1}$, indicating $\omega_{\text {rel }}=6.3 \mathrm{rad} \mathrm{s}^{-1}$, which is in good agreement with the experimental value $6 \mathrm{rad} \mathrm{s}^{-1}$. The value of $f_{2}$ should be determined by measuring the semiaxes length. At present, unfortunately, it is difficult because our blend is turbid. The direct observation is our future plan.

At the end of this paper, we calculate the stress response to ac electric fields in order to compare the experimental result with the theory. We add a term to Eq. (7) as a driving force to deform a droplet

$$
c[\mathbf{E E}-h(\mathbf{S}, \mathbf{E}) \mathbf{I}],
$$

with

$$
h(\mathbf{S}, \mathbf{E})=\sum_{i, j, k, l, m, n} \varepsilon_{i j k} \varepsilon_{l m n} E_{i} E_{l} S_{j m} S_{k n} / 2 I_{2}(\mathbf{S}),
$$

where $h(\mathbf{S}, \mathbf{E})$ is introduced to preserve the droplet volume, $\varepsilon_{i j k}$ is the permutation tensor, and $c$ is a constant. Adding this term to Eq. (7), we obtain the deformation parameter $D$ $=(L-B) /(L+B)$ without shear flow for a small dc electric field $E$ as

$$
D=\frac{c}{4 \tau R^{2} f_{1}} E^{2} .
$$

The constant $c$ should be determined so that the above equation coincides with the results of theories, giving the droplet deformation under electric fields. For example, for perfect dielectrics, i.e., $\kappa_{1}=\kappa_{2}=0[13,25], c$ is given as

$$
c=\frac{9 f_{1} \varepsilon_{2}\left(\varepsilon_{1}-\varepsilon_{2}\right)^{2} R^{2}}{4 \eta_{2}\left(\varepsilon_{1}+2 \varepsilon_{2}\right)^{2}} .
$$

From Eqs. (7) and (30), we can calculate the response of deformation when subjected to ac electric fields and, furthermore, the corresponding shear stress tensor by utilizing the formula [8]

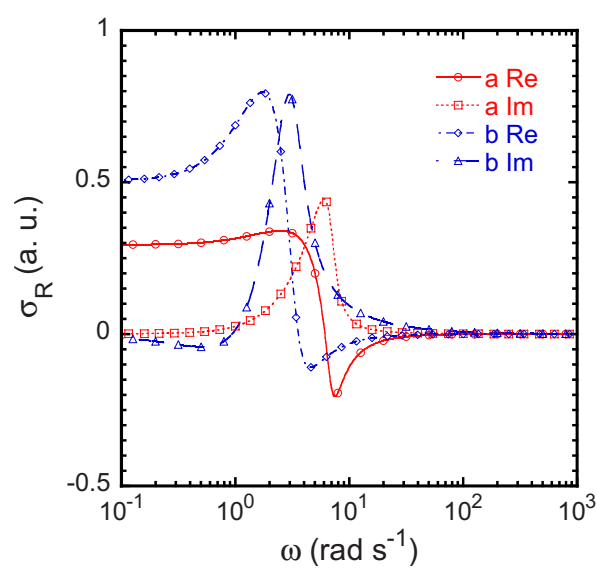

FIG. 8. (Color online) Frequency dispersion of $\sigma_{R}$ at (a) $f_{1} / \tau$ $=2.5 \mathrm{rad} \mathrm{s}^{-1}, f_{2}=0.725, \dot{\gamma}=20 \mathrm{~s}^{-1}$, and (b) $f_{1} / \tau=2.5 \mathrm{rad} \mathrm{s}^{-1}, f_{2}$ $=0.9, \dot{\gamma}=14 \mathrm{~s}^{-1}$. The shear stress was calculated for the parallel plates corresponding to the experimental results by using Eq. (35).

$$
\sigma^{s}=\frac{2 f_{2} K}{I_{2}}\left(I_{1} \mathbf{S}-\mathbf{S} \cdot \mathbf{S}-\frac{2}{3} I_{2} \mathbf{I}\right)
$$

with

$$
K=\frac{6 \Gamma}{5 R} \frac{(\lambda+1)(2 \lambda+3) \varphi}{5(\lambda+1)-(5 \lambda+2) \varphi},
$$

where $\varphi$ is the volume fraction of droplets. Figure 8 shows the frequency dispersion of $\sigma_{12}^{s}$ for two cases of parameters (a) $f_{1} / \tau=2.5 \mathrm{rad} \mathrm{s}^{-1}, f_{2}=0.725, \dot{\gamma}=20 \mathrm{~s}^{-1}$, and (b) $f_{1} / \tau$ $=2.5 \mathrm{rad} \mathrm{s}^{-1}, f_{2}=0.9, \dot{\gamma}=14 \mathrm{~s}^{-1}$, where we calculated the shear stress for the parallel plates corresponding to the experimental result by using the following equation:

$$
\sigma_{R}=4 \dot{\gamma}_{R}^{-3} \int_{0}^{\dot{\gamma}_{R}} \sigma^{s}(\dot{\gamma}) \dot{\gamma}^{2} d \dot{\gamma},
$$

where $\dot{\gamma}_{R}$ is the shear rate at the edge of the rotating disk and $\sigma_{R}$ is the shear stress calculated by assuming that the blend is Newtonian. Note that the scale of the ordinate is arbitrary because $R$ and $\Gamma$ were not independently determined in our experiments. The negative real part is successfully reproduced both in the cases. On the other hand, the peak frequencies of the imaginary part are 6 and $3 \mathrm{rad} \mathrm{s}^{-1}$ for the cases (a) and (b), respectively. The latter case, in which the hydrodynamic effect was taken into account and another choice of $f_{2}=0.9$ was adopted, coincides well with the experiment. Comparing the experiment (Fig. 3) and calculated results (Fig. 8) in more detail, it is noticed that there is another lower mode in the experiment. This mode may be ascribed to the translational motion of droplets to form chains along the applied electric field.

\section{SUMMARY}

We measured the response of shear stress to ac electric fields to investigate the dynamics of a droplet-dispersed phase under steady shear flow. A scaling property derived from dimensional analysis was confirmed to almost hold in 
the experiment. It was found that the real part of the response becomes negative under steady shear flow at large shear rates, which may be a remarkable feature characteristic to our nonequilibrium steady system. The negative real part was clarified to be due to the normal modes with complex eigenvalues in the MM model. Also for the superimposed storage modulus measured by Chen et al. [6], it is easily shown that it becomes negative by calculations using complex eigenvalues. The MM model was modified to calculate the frequency dependence of the stress and a good agreement was obtained with the experiment except at low frequencies.

\section{ACKNOWLEDGMENTS}

We thank Professor T. Ohta and Dr. T Sakaue for useful discussions. This work was partially supported by Grant-inAid for Scientific Research [Priority Area "Soft Matter Physics" (Contract No. 463), C (Contract No. 19540326), and Wakate B (Contract No. 19740255)] from the Ministry of Education, Culture, Sports, Science and Technology of Japan.

\section{APPENDIX}

The electric force added to Eq. (7) should be a secondrank tensor as well as $\mathbf{S}$. The lowest-order term of the force with respect to $\mathbf{E}$ may be written in the simplest form as Eq. (29). $h(\mathbf{S}, \mathbf{E})$ can be obtained from the constant volume condition as follows. We consider the equation of motion, including Eq. (29). When a static electric field is applied along the $x_{1}$ axis without flow, the equation for each of the three eigenvalues $S_{i}$ of $\mathbf{S}$ becomes

$$
\begin{gathered}
\frac{d S_{1}}{d t}=-\frac{f_{1}}{\tau}\left[S_{1}-g(\mathbf{S})\right]+c\left[E_{1}^{2}-h(\mathbf{S}, \mathbf{E})\right], \\
\frac{d S_{2}}{d t}=-\frac{f_{1}}{\tau}\left[S_{2}-g(\mathbf{S})\right]-\operatorname{ch}(\mathbf{S}, \mathbf{E}), \\
\frac{d S_{3}}{d t}=-\frac{f_{1}}{\tau}\left[S_{3}-g(\mathbf{S})\right]-\operatorname{ch}(\mathbf{S}, \mathbf{E}),
\end{gathered}
$$

where we have assumed that the eigenvector corresponding to the eigenvalue $S_{1}$ is parallel to the field direction. Multiplying the first, second and third equations by $S_{2} S_{3}, S_{1} S_{3}$, and $S_{1} S_{2}$, respectively, and summing up them, we obtain

$$
\begin{aligned}
\frac{d}{d t}\left(S_{1} S_{2} S_{3}\right)= & -\frac{f_{1}}{\tau}\left[3 S_{1} S_{2} S_{3}-\left(S_{1}+S_{2}+S_{3}\right) g(\mathbf{S})\right]+c\left[E_{1}^{2} S_{2} S_{3}\right. \\
& \left.-\left(S_{1} S_{2}+S_{2} S_{3}+S_{3} S_{1}\right) h(\mathbf{S}, \mathbf{E})\right] .
\end{aligned}
$$

The left-hand side is zero because $S_{1} S_{2} S_{3}$ is the square of the volume, and the first term of the right-hand side also vanishes from Eq. (13). Thus, $h(\mathbf{S}, \mathbf{E})$ is given as

$$
h(\mathbf{S}, \mathbf{E})=\frac{E_{1}^{2} S_{2} S_{3}}{S_{1} S_{2}+S_{2} S_{3}+S_{3} S_{1}} .
$$

This implies Eq. (30), which was generally confirmed by the algebraic computation.
[1] C. L. Tucker and P. Moldenaers, Annu. Rev. Fluid Mech. 34, 177 (2002).

[2] J. F. Palierne, Rheol. Acta 29, 204 (1990); 30, 497(E) (1991).

[3] D. Graebling and R. Muller, J. Rheol. 34, 193 (1990).

[4] D. Graebling, R. Muller, and J. F. Palierne, Macromolecules 26, 320 (1993).

[5] J. Vermant, L. Walker, P. Moldenaers, and J. Mewis, J. NonNewtonian Fluid Mech. 79, 173 (1998).

[6] Q. Chen, W. Yu, and C. Zhou, J. Polym. Sci., Part B: Polym. Phys. 46, 431 (2008).

[7] P. L. Maffettone and M. Minale, J. Non-Newtonian Fluid Mech. 78, 227 (1998); 84, 105(E) (1999).

[8] W. Yu, M. Bousmina, M. Grmela, and C. J. Zhou, J. Rheol. 46, 1401 (2002).

[9] T. Sakaue and T. Ohta, Phys. Rev. E 77, 050102(R) (2008).

[10] T. Ohta and T. Ohkuma, J. Phys. Soc. Jpn. 77, 074004 (2008).

[11] P. Panizza, L. Courbin, G. Cristobal, J. Rouch, and T. Narayanan, Physica A 322, 38 (2003); L. Courbin, P. Panizza, and J.-B. Salmon, Phys. Rev. Lett. 92, 018305 (2004); L. Courbin, A. Benayad, and P. Panizza, Phys. Rev. E 73, 011501 (2006).

[12] M. Grosso, S. Crescitelli, E. Somma, J. Vermant, P. Moldenaers, and P. L. Maffettone, Phys. Rev. Lett. 90, 098304 (2003); M. Grosso, R. Keunings, S. Crescitelli, and P. L. Ma- ffettone, ibid. 86, 3184 (2001).

[13] S. Torza, R. G. Cox, and S. G. Mason, Philos. Trans. R. Soc. London, Ser. A 269, 295 (1971).

[14] E. Lac and G. M. Homsy, J. Fluid Mech. 590, 239 (2007).

[15] A. Inoue, Y. Ide, and H. Oda, J. Appl. Polym. Sci. 64, 1319 (1997)

[16] K. Tajiri, H. Orihara, Y. Ishibashi, M. Doi, and A. Inoue, J. Rheol. 43, 137 (1999).

[17] Y.-H. Na, T. Shibuya, S. Ujiie, T. Nagaya, and H. Orihara, Phys. Rev. E 77, 041405 (2008).

[18] H. Orihara, T. Shibuya, T. Nagaya, and S. Ujiie, J. Phys. Soc. Jpn. 75, 063802 (2006)

[19] H. Orihara, K. Tanabu, and Y. H. Na, J. Phys. Soc. Jpn. 77, 093802 (2008).

[20] M. Doi and T. Ohta, J. Chem. Phys. 95, 1242 (1991).

[21] M. Parthasarathy and D. J. Klingenberg, Mater. Sci. Eng. R. 17, 57 (1996).

[22] R. G. Cox, J. Fluid Mech. 37, 601 (1969).

[23] N. A. Frankel and A. Acrivos, J. Fluid Mech. 44, 65 (1970).

[24] S. Torquato, B. Lu, and J. Rubinstein, Phys. Rev. A 41, 2059 (1990).

[25] R. S. Allan and S. G. Mason, Proc. R. Soc. London, Ser. A Math. Phys. Sci. 267, 45 (1962). 